\title{
Video Article \\ Direct Delivery of MIF Morpholinos Into the Zebrafish Otocyst by Injection and Electroporation Affects Inner Ear Development
}

\author{
Katie E. Holmes ${ }^{1}$, Matthew J. Wyatt ${ }^{2}$, Yu-chi Shen ${ }^{2}$, Deborah A. Thompson ${ }^{2,3}$, Kate F. Barald ${ }^{2,4}$ \\ ${ }^{1}$ Department of Veterinary Science, University of Wisconsin, Madison \\ ${ }^{2}$ Department of Cell and Developmental Biology, University of Michigan, Ann Arbor, MI \\ ${ }^{3}$ Present address: Department of Pulmonary Medicine, University of Michigan, Ann Arbor, MI \\ ${ }^{4}$ Department of Biomedical Engineering, University of Michigan, Ann Arbor, MI
}

Correspondence to: Kate F. Barald at kfbarald@umich.edu

URL: https://www.jove.com/video/2466

DOI: doi:10.3791/2466

Keywords: Developmental Biology, Issue 47, Zebrafish inner ear, microinjection, electroporation, morpholino

Date Published: 1/7/2011

Citation: Holmes, K.E., Wyatt, M.J., Shen, Y.c., Thompson, D.A., Barald, K.F. Direct Delivery of MIF Morpholinos Into the Zebrafish Otocyst by Injection and Electroporation Affects Inner Ear Development. J. Vis. Exp. (47), e2466, doi:10.3791/2466 (2011).

\section{Abstract}

In recent years, electroporation has become a popular technique for in vivo transfection of DNA, RNA, and morpholinos into various tissues, including the eye, brain, and somites of zebrafish. The advantage of electroporation over other methods of genetic manipulation is that specific tissues can be targeted, both spatially and temporally, for the introduction of macromolecules by the application of electrical current. Here we describe the use of electroporation for transfecting mif and mif-like morpholinos into the tissues of the developing inner ear of the zebrafish. In past studies, mif morpholino injected into embryos at the 1- to 8-cell stage resulted in widespread morphological changes in the nervous system and eye, as well as the ear. By targeting the tissues of the inner ear at later stages in development, we can determine the primary effects of MIF in the developing inner ear, as opposed to secondary effects that may result from the influence of other tissues. By using phalloidin and acetylated tubulin staining to study the morphology of neurons, neuronal processes, and hair cells associated with the posterior macula, we were able to assess the efficacy of electroporation as a method for targeted transfection in the zebrafish inner ear. The otic vesicles of $24 \mathrm{hpf}$ embryos were injected with morpholinos and electroporated and were then compared to embryos that had received no treatment or had been only injected or electroporated. Embryos that were injected and electroporated showed a decrease in hair cell numbers, decreased innervation by the statoacoustic ganglion (SAG) and fewer SAG neurons compared with control groups. Our results showed that direct delivery of morpholinos into otocysts at later stages avoids the non-specific nervous system and neural crest effects of morpholinos delivered at the 1-8 cell stage. It also allows examination of effects that are directed to the inner ear and not secondary effects on the ear from primary effects on the brain, neural crest or periotic mesenchyme.

\section{Video Link}

The video component of this article can be found at https://www.jove.com/video/2466/

Protocol

\section{Making Electrodes for Electroporation}

1. Cut $75 \mu \mathrm{m}$ diameter tungsten wire (A-M Systems, Inc. Carlsborg, WA) of suitable length (about $3-5 \mathrm{~cm}$ )

2. Put tungsten wire through molex cable connector (pin stamped brass)

3. Wrap tungsten wires and molex cable connector with shrink tubing (SPC Technology, Chicago, IL) and apply heat with Bunsen Burner or heat gun to seal the shrinking tubing to the wire

4. To sharpen the tip of tungsten wire, dip the wire into $1.0 \mathrm{~N}$ Sodium Hydroxide (Conrad et al. 1993) and, using a paper-clip as the other electrode, electrolyze the wire with a Square Wave Electroporator. The conditions we use are 550 -Volt pulses each lasting $100 \mathrm{~ms}$ with 50 $\mathrm{ms}$ in between pulses. Electrodes should be sharpened to a diameter of 15-20 $\mu \mathrm{m}$

5. Keep the wires in a tray within grooves pressed into modelling clay prior to use in electroporation

\section{Set Up Electroporation Station}

1. Tape sharpened $75 \mu \mathrm{m}$ tungsten electrodes to micromanipulators (World Precision instruments).

2. Place micromanipulators on either side of the dissecting microscope stage (Leica).

3. Connect the electrodes to a Protect CUY-21 Edit Square Wave Electroporator (Figure 1). 
4. Turn on the electroporator and set up Parameters for electroporation, including voltage, pulse duration, and number of pulses. The most effective parameters for this experiment were found to be three 13-volt pulses which each lasted for $5.0 \mathrm{~ms}$, with $100 \mathrm{~ms}$ in between each pulse. If electroporation creates air bubbles in the embryo, reduce the pulse length.

\section{Microinjection of Morpholinos Into Zebrafish Otic Vesicle}

1. Harvest zebrafish embryos from a breeding tank. Incubate the embryos in embryo-raising medium with 0.3 PPM methylene blue (Westerfield, 2005) at $28.5^{\circ} \mathrm{C}$ overnight.

2. Pull glass needles with a Sutter P-97 electrode puller

3. Warm up an agarose gel base (1\% agarose in fish water in a $10 \mathrm{~mm}$ Petri dish) to $37^{\circ} \mathrm{C}$ in a water bath

4. Heat $1 \%$ low melting point agarose (LMPA) in fish water until melted and keep it warm in the $37^{\circ} \mathrm{C}$ water bath.

5. Fill a glass needle with a morpholino solution (GeneTools, Corvallis, OR) and cut the tip to appropriate diameter. Mount the injection needle onto a micromanipulator (Kuhn).

6. Cut needle tip to approximately $10 \mu \mathrm{m}$ and inject into mineral oil to ensure that the tip allows sufficient delivery of morpholino solution.

7. Dechorinate embryos at 24 hours postfertilization (hpf) and anesthetize them with MS222 (tricaine, Sigma) in 0.3 PPM methylene blue fish water.

8. Align 3 to 5 anesthetized embryos onto the agarose gel base with the right side up for convenient uniform analysis

9. Put 1 to 2 drops of $1 \%$ LMPA over each embryo and let solidify to fix the embryo in place

10. Rotate the Petri dish so that the otic vesicle is on the right side

11. Place the needle into the lumen of the otic vesicle and inject the morpholino solution with a PV820 Pneumatic PicoPump (World Precision Instruments) with an attached nitrogen tank (Figure 2)

\section{Electroporation Procedure}

1. Move the mounted embryos to the electroporation station immediately after injection

2. Place the positive electrode onto the tissue just posterior to the otic vesicle, but do not penetrate; insert the negative electrode into the brain just anterior to the otic vesicle

3. Apply current using a foot pedal or by pushing a switch.

4. Pour fish water on agarose and remove embryos from agarose with swirling and a glass pipette. Use caution not to damage the embryos. For embryos that are difficult to remove, use a needle to gently break away any LMPA surrounding the embryo.

5. Place embryos in a plate with methylene blue fish water and raise the embryos to desired stages for analysis (morphological, immunohistochemical, in situ hybridization, etc and microscopic anlaysis).

\section{Representative Results:}

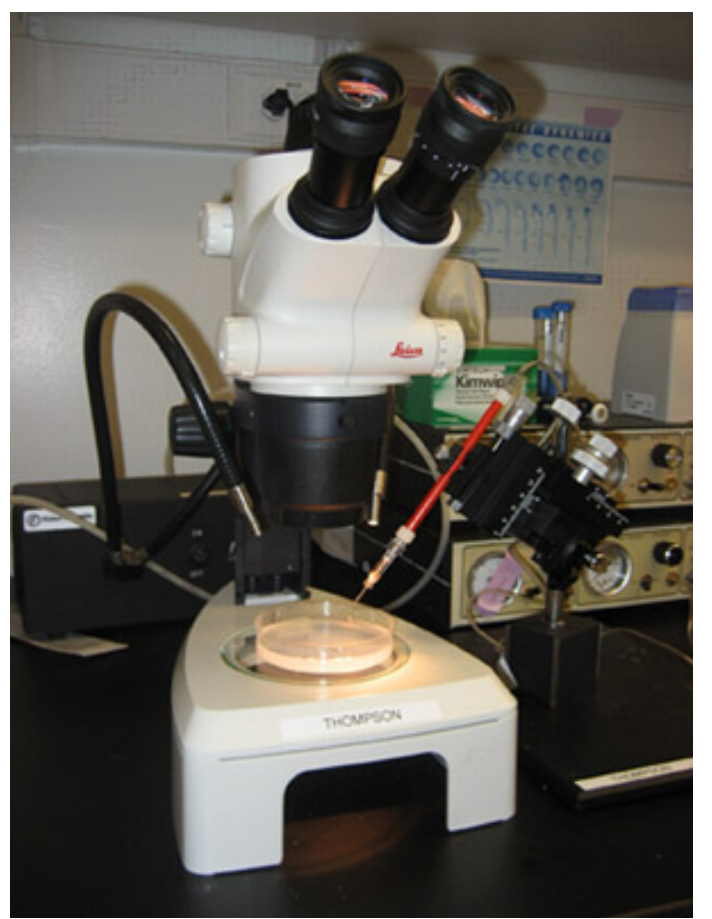

Figure 1. Electroporation station. Electrodes were made using sharpened $75 \mu \mathrm{m}$ Tungsten wires and connected by leads to a Protect CUY-21 Edit Square Wave Electroporator. These electrodes were taped to micromanipulators. 

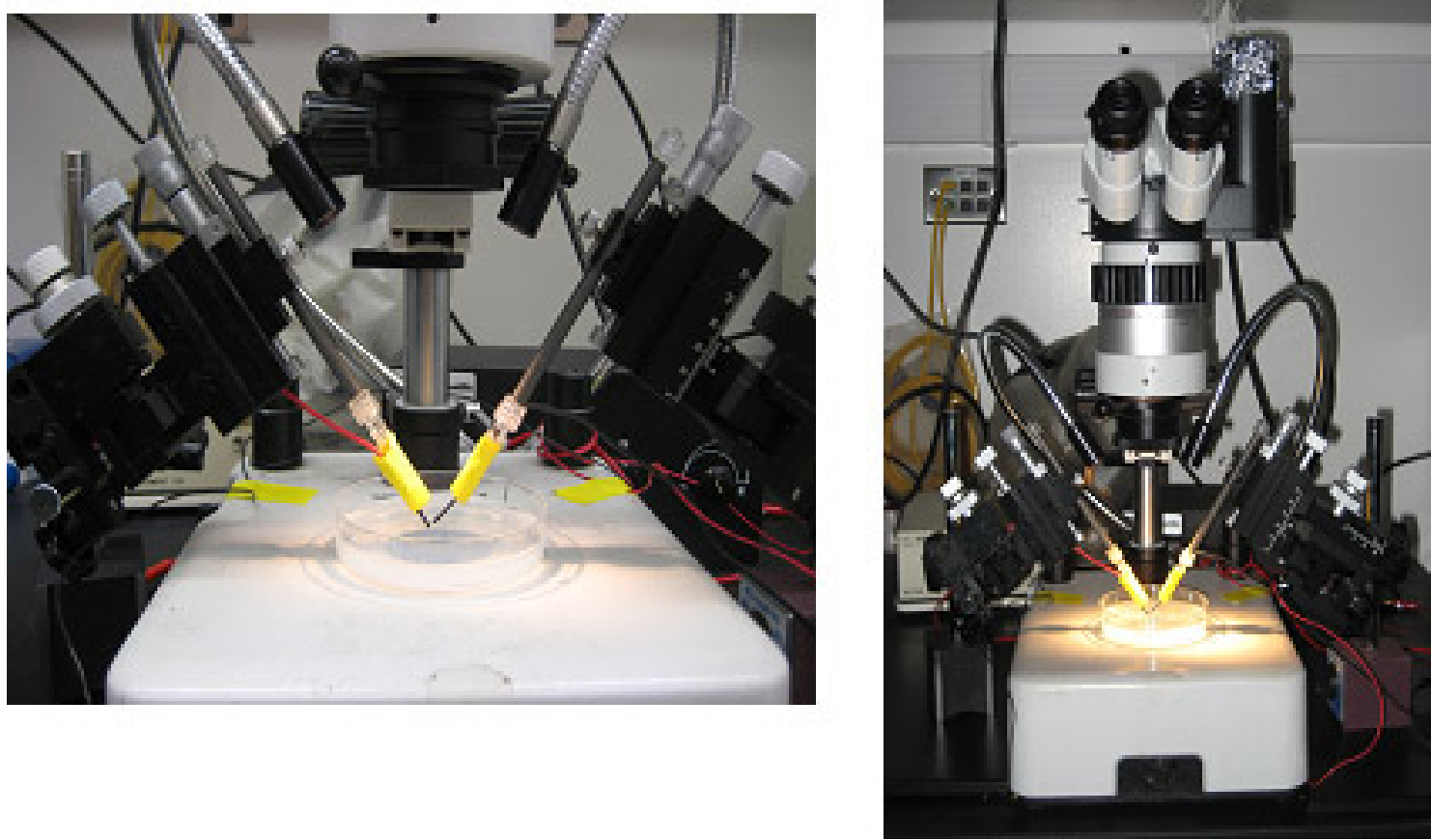

Figure 2. Injection station. All embryos were injected into the right ear for standardization purposes.

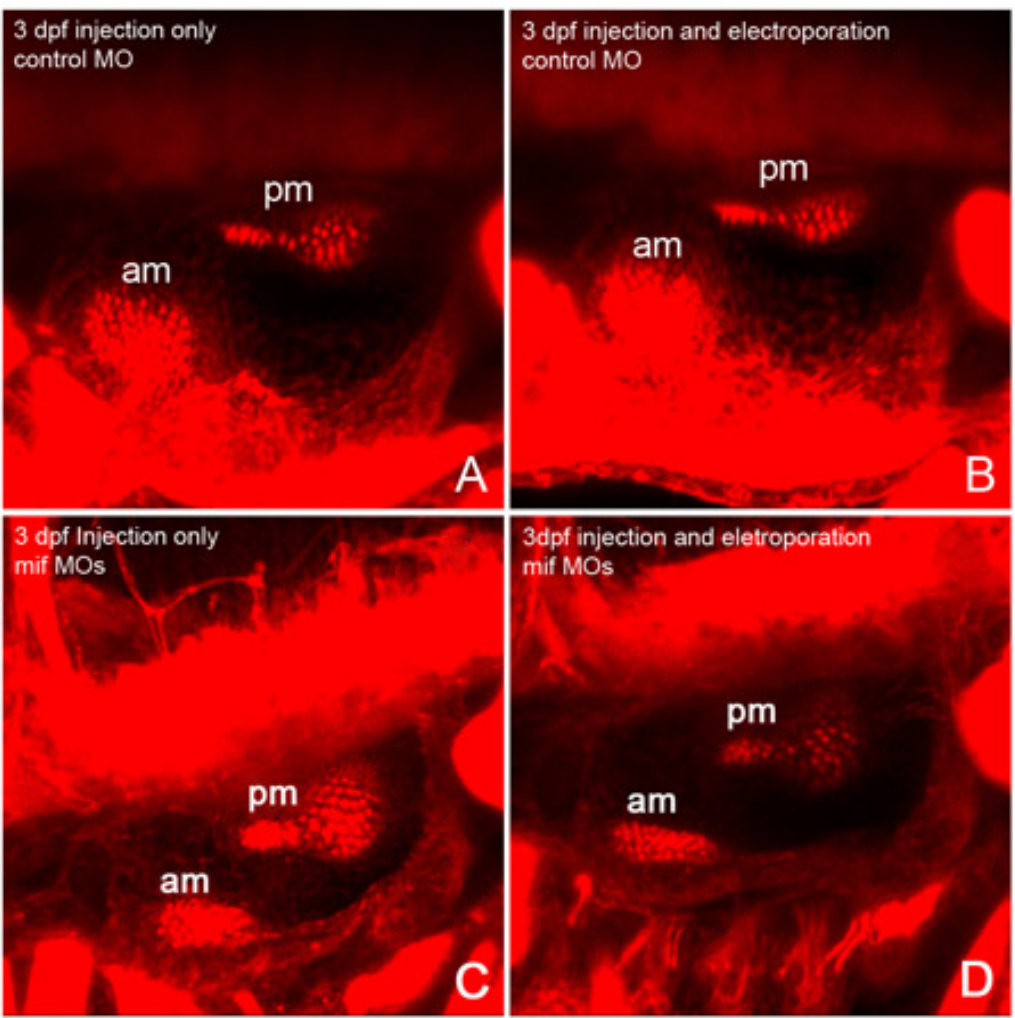

Figure 3. Phalloidin staining of actin filaments with $3 \mathrm{dpf}$ embryos. (A) The 3 dpf embryo injected with control MO has strong staining of phalloidin in the posterior macula (pm). (B) The embryo which was injected with control MO and then electroporated had similar levels of phalloidin staining in pm in the injection only embryos. (C) Injection with mif MOs into the otic vesicle alone did not cause reduction of phalloidin staining, while injection of mif MOs along with electroporation caused a dramatic reduction of phalloidin staining in the pm (D). am, anterior macula. 


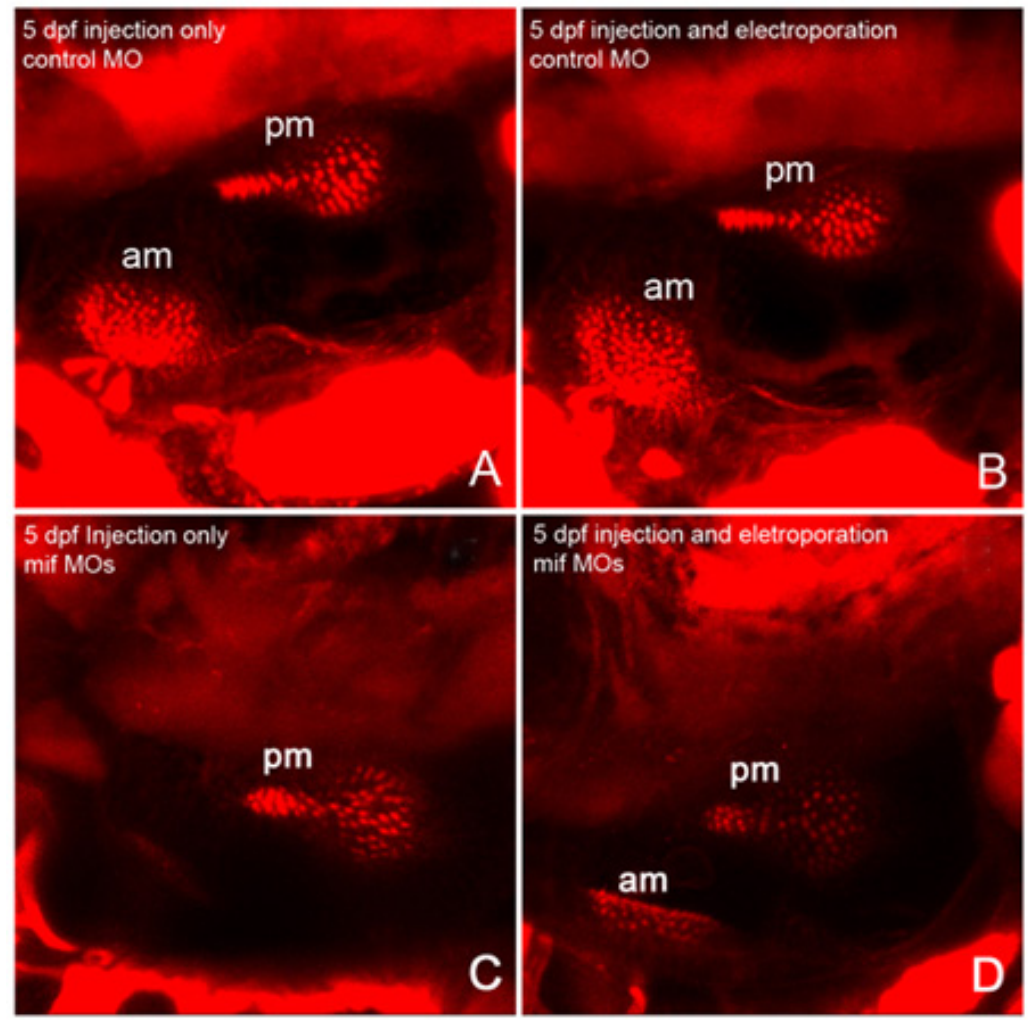

Figure 4. Phalloidin staining of actin filaments with $5 \mathrm{dpf}$ larvae. There was no difference between injection only (A) and injection with electroporation when the standard control morpholino was used (B). However, the $5 \mathrm{dpf}$ larvae that were injected with mif morpholinos and electroporated showed reduced phalloidin staining in the posterior macula $(\mathrm{pm})(\mathrm{D})$, though less dramatic than $3 \mathrm{dpf}$, when compared with the embryo that was injected with mif morpholinos only (C). am, anterior macula.

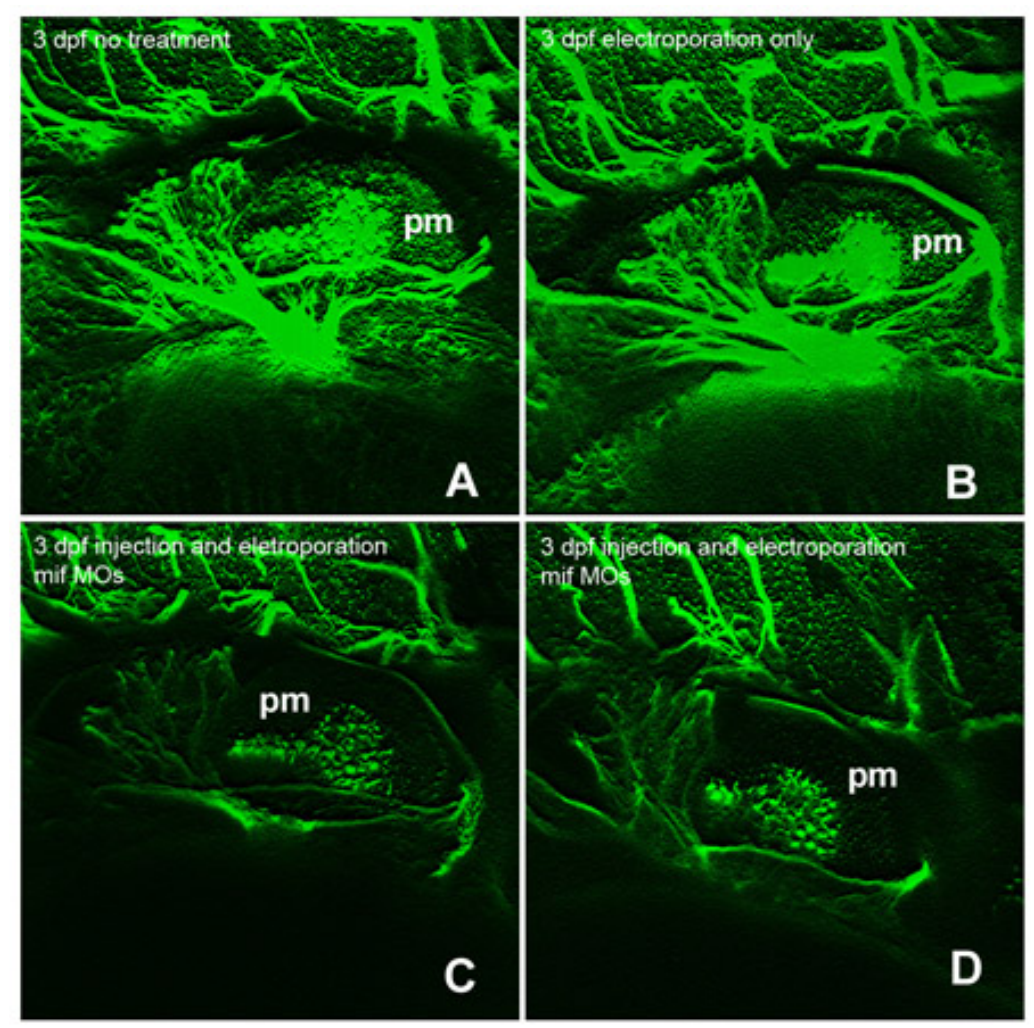


Figure 5. Acetylated tubulin staining of embryos at $3 \mathrm{dpf}$. The embryo in (A) did not receive any injection or electroporation. The embryo in (B) was electroporated but received no injection. Embryos receiving both injection and electroporation (C, D) with mif morpholinos had visibly diminished tubulin staining compared to controls. (pm, posterior macula).
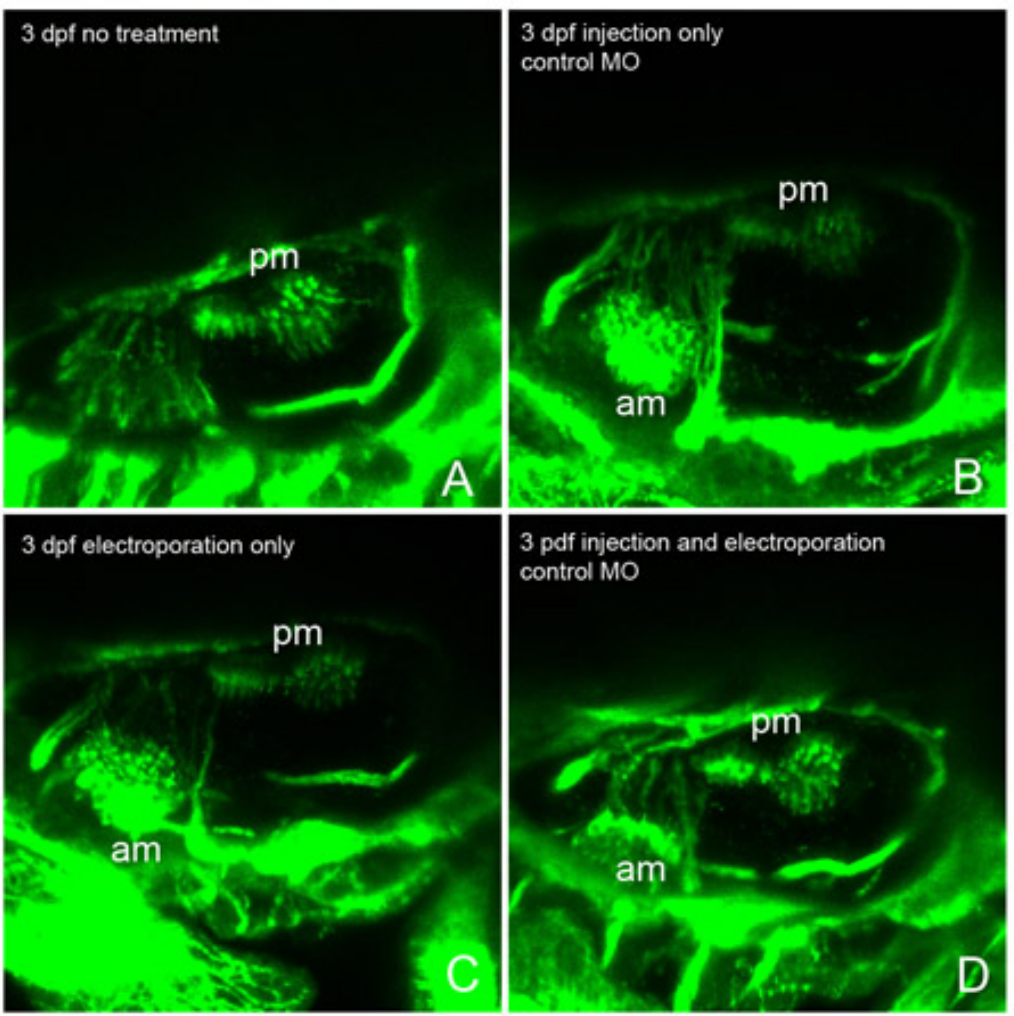

Figure 6. Acetylated tubulin staining of embryos at $3 \mathrm{dpf}$ with control morpholino. (A) Embryo without any treatment showed strong acetylated tubulin staining in the posterior macula $(\mathrm{pm})$ and extensive innervation. (B) Embryo injected with control morpholino. (C) Embryo treated with electroporation only. (D) Control embryo with control morpholino injected and electroporated has similar levels of acetylated tubulin in the posterior macula and innervation compared to the no treatment control.

Discussion

We have successfully introduced antisense oligonucleotide MOs into the ear of the $24 \mathrm{hpf}$ zebrafish embryo. Embryos that were raised after injection and electroporation were viable. If the embryos survived the electroporation, they grew at normal rates compared to embryos that had received no treatment, as well as embryos that had only been injected and embryos that had only been electroporated.

We observed the effects of mif and mif-like MOs after ear injection and electroporation through labeling with phalloidin and acetylated tubulin. Phalloidin staining of actin filaments in sensory hair cells of the posterior macula indicates that electroporation of mif and mif-like MO at the 24hpf stage caused changes in hair cell and/or stereocilia numbers when compared to embryos that were only injected with the MOs (Figure 3 ). This decrease in hair cell numbers is consistent with the findings of Shen et al. (submitted) that MOs to mif and mif-like cause a reduction in the number of hair cells in the saccular macula. The decrease in hair cell numbers in embryos that were injected with MOs and electroporated demonstrate that electroporation may aid in moving the MOs across the cell membrane, thereby increasing the extent of the morphological effects when compared to embryos in which the otic vesicle was only injected. Changes in the morphology of the statoacoustic ganglion were observed through acetylated tubulin staining. The extent of innervation by the statoacoustic ganglion was affected, as embryos that were injected and electroporated show decreased branching of the neurites (Figure 5). There may also be decreased condensation of the ganglionic cells and/or decreased number of ganglion cells, because the acetylated tubulin staining is significantly decreased in embryos that were both injected and electroporated. Because mif has been shown to be critical for nervous system development (Suzuki et al., 2004), the changes seen after electroporation may be the result of increased transfection of the mif and mif-like MO solution into the neuroblast cells.

Electroporation of antisense oligo morpholinos directly into a developing tissue is a useful tool for controlling the expression of a gene during that tissue's development, both spatially and temporally, in the embryo. Electroporation of mif and mif-like MOs into the tissues of the inner ear resulted in abnormal morphology of both the posterior macula and the statoacoustic ganglion. There was a reduction in the number of sensory hair cells in the posterior macula in embryos that had been injected and electroporated in comparison with embryos that had received no treatment or with embryos that had either been only injected or only electroporated. There was also a decrease in the degree of innervation of the posterior sensory patch by the statoacoustic ganglion and the size of the SAG. Electroporation is a valuable method for transfecting macromolecules into specific tissues, with the benefit of observing the primary effects of that particular DNA, RNA, or MO in the desired tissue and discriminating these from secondary effects on other tissues, including the brain, neural crest and periotic mesenchyme on inner ear development (Barald and Kelley, 2004). 


\section{Disclosures}

The production of the article was sponsored by Gene Tools, LLC who produce reagents mentioned in the video and text.

\section{Acknowledgements}

This work was supported by grants from the Deafness Research Foundation to Y-c S and the NSF (IOS 0930096) to KFB.

KFB: NIH/NINDCD 2 RO1 DC04184, 3R01DC004184-08W1, NSF DBI 0832862, NSF IOS 0930096

\section{References}

1. Conrad, G.W., Bee, J.A., Roche, S.M., \& Teillet, M.A. Fabrication of microscalpels by electrolysis of tungsten wire in a meniscus. J Neurosci Methods 50 (1): 123-7 (1993).

2. Suzuki, M., Takamura, Y., Maeno, M., Tochinai, S., Iyaguchi, D., Tanaka, I., Nishihira, J., Ishibashi, T. Xenopus laevis macrophage migration inhibitory factor is essential for axis formation and neural development. J Biol Chem 279 : 21406-21414 (2004).

3. Barald, K.F., \& Kelley, M.W. From placode to polarization: new tunes in inner ear development. Development 131, 4119-4135 (2004). 